Honam Mathematical J. 34 (2012), No. 2, pp. 135-144

http://dx.doi.org/10.5831/HMJ.2012.34.2.135

\title{
FALLING SHADOWS APPLIED TO SUBALGEBRAS AND IDEALS OF BCK/BCI-ALGEBRAS
}

\author{
Young Bae Jun and Chul Hwan ParK*
}

\begin{abstract}
BCK/BCI-algebra is introduced. Relations between falling subalgebras and falling ideals are given. Relations between fuzzy subalgebras/ideals and falling subalgebras/ideals are provided. A characterization of a falling ideal is established.
\end{abstract}

\section{Introduction and Preliminaries}

\subsection{Introduction}

In the study of a unified treatment of uncertainty modelled by means of combining probability and fuzzy set theory, Goodman [2] pointed out the equivalence of a fuzzy set and a class of random sets. Wang and Sanchez [8] introduced the theory of falling shadows which directly relates probability concepts with the membership function of fuzzy sets. The mathematical structure of the theory of falling shadows is formulated in [7]. Tan et al. [5, 6] established a theoretical approach to define a fuzzy inference relation and fuzzy set operations based on the theory of falling shadows. Yuan and Lee [9] considered a fuzzy subgroup (subring, ideal) as the falling shadow of the cloud of the subgroup (subring, ideal). In this article, we introduce the notion of falling subalgebras/ideals in $\mathrm{BCK} / \mathrm{BCI}$-algebras based on the theory of falling shadows. We give relations between falling subalgebras and falling ideals. We also provide relations between fuzzy subalgebras/ideals and falling subalgebras/ideals. We establish a characterization of a falling ideal. We show that every falling subalgebra/ideal is a $T_{m}$-fuzzy subalgebra/ideal.

Received August 25, 2011. Accepted April 12, 2012.

2000 Mathematics Subject Classification. 06F35, 03G25, 08 A72. ideal.

Key words and phrases. Falling shadow, Cloud, (falling) subalgebra, (falling)

*Corresponding author. 


\subsection{Basic results on BCK/BCI-algebras and fuzzy aspects}

A BCK/ BCI-algebra is an important class of logical algebras introduced by K. Iséki and was extensively investigated by several researchers.

An algebra $(X ; *, 0)$ of type $(2,0)$ is called a $B C I$-algebra if it satisfies the following conditions:

(I) $(\forall x, y, z \in X)(((x * y) *(x * z)) *(z * y)=0)$,

(II) $(\forall x, y \in X)((x *(x * y)) * y=0)$,

(III) $(\forall x \in X)(x * x=0)$,

(IV) $(\forall x, y \in X)(x * y=0, y * x=0 \Rightarrow x=y)$.

If a BCI-algebra $X$ satisfies the following identity:

(V) $(\forall x \in X)(0 * x=0)$,

then $X$ is called a $B C K$-algebra. Any BCK/BCI-algebra $X$ satisfies the following axioms:

(a1) $(\forall x \in X)(x * 0=x)$,

(a2) $(\forall x, y, z \in X)(x \leq y \Rightarrow x * z \leq y * z, z * y \leq z * x)$,

(a3) $(\forall x, y, z \in X)((x * y) * z=(x * z) * y)$,

where $x \leq y$ if and only if $x * y=0$. A nonempty subset $S$ of a BCK/BCIalgebra $X$ is called a subalgebra of $X$ if $x * y \in S$ for all $x, y \in S$.

A subset $I$ of a BCK/BCI-algebra $X$ is called an ideal of $X$, denoted by $I \triangleleft X$, if it satisfies:

(i) $0 \in I$

(ii) $(\forall x \in X)(\forall y \in I)(x * y \in I \Longrightarrow x \in I)$.

Every ideal $I$ of a BCK/BCI-algebra $X$ has the following assertion:

$$
(\forall x \in X)(\forall y \in I)(x \leq y \Longrightarrow x \in I) .
$$

We refer the reader to the paper [3] and book [4] for further information regarding $\mathrm{BCK} / \mathrm{BCI}$-algebras.

A fuzzy set $\mu$ in a BCK/BCI-algebra $X$ is called a fuzzy subalgebra of $X$ if it satisfies:

$$
(\forall x, y \in X)(\mu(x * y) \geq \min \{\mu(x), \mu(y)\}) .
$$

A fuzzy set $\mu$ in a BCK/BCI-algebra $X$ is called a fuzzy ideal of $X$ if it satisfies:

(i) $(\forall x \in X)(\mu(0) \geq \mu(x))$.

(ii) $(\forall x, y \in X)(\mu(x) \geq \min \{\mu(x * y), \mu(y)\})$.

Proposition 1.1. Let $\mu$ be a fuzzy set in a BCK/BCI-algebra $X$. Then $\mu$ is a fuzzy ideal of $X$ if and only if

$$
(\forall t \in[0,1])\left(\mu_{t}:=\{x \in X \mid \mu(x) \geq t\} \triangleleft X\right) .
$$




\subsection{The Theory of Falling Shadows}

Given a universe of discourse $U$, let $\mathscr{P}(U)$ denote the power set of $U$. For each $u \in U$, let

$$
\dot{u}:=\{E \mid u \in E \text { and } E \subseteq U\} .
$$

For each $E \in \mathscr{P}(U)$, let

$$
\dot{E}:=\{\dot{u} \mid u \in E\} .
$$

An ordered pair $(\mathscr{P}(U), \mathscr{B})$ is said to be a hyper-measurable structure on $U$ if $\mathscr{B}$ is a $\sigma$-field in $\mathscr{P}(U)$ and $\dot{U} \subseteq \mathscr{B}$. Given a probability space $(\Omega, \mathscr{A}, P)$ and a hyper-measurable structure $(\mathscr{P}(U), \mathscr{B})$ on $U$, a random set on $U$ is defined to be a mapping $\xi: \Omega \rightarrow \mathscr{P}(U)$ which is $\mathscr{A}-\mathscr{B}$ measurable, that is,

$$
(\forall C \in \mathscr{B})\left(\xi^{-1}(C)=\{\omega \mid \omega \in \Omega \text { and } \xi(\omega) \in C\} \in \mathscr{A}\right) .
$$

Suppose that $\xi$ is a random set on $U$. Let

$$
\tilde{H}(u):=P(\omega \mid u \in \xi(\omega)) \text { for each } u \in U .
$$

Then $\tilde{H}$ is a kind of fuzzy set in $U$. We call $\tilde{H}$ a falling shadow of the random set $\xi$, and $\xi$ is called a cloud of $\tilde{H}$.

For example, $(\Omega, \mathscr{A}, P)=([0,1], \mathscr{A}, m)$, where $\mathscr{A}$ is a Borel field on $[0,1]$ and $m$ the usual Lebesgue measure. Let $\tilde{H}$ be a fuzzy set in $U$ and $\tilde{H}_{t}:=\{u \in U \mid \tilde{H}(u) \geq t\}$ be a $t$-cut of $\tilde{H}$. Then

$$
\xi:[0,1] \rightarrow \mathscr{P}(U), t \mapsto \tilde{H}_{t}
$$

is a random set and $\xi$ is a cloud of $\tilde{H}$. We shall call $\xi$ defined above as the cut-cloud of $\tilde{H}$ (see [2]).

\section{Fuzzy subalgebras/ideals based on the theory of falling shadows}

Definition 2.1. Let $X$ be a BCK/BCI-algebra, $(\Omega, \mathscr{A}, P)$ a probability space, and let

$$
\xi: \Omega \rightarrow \mathscr{P}(X)
$$

be a random set. If $\xi(\omega)$ is a subalgebra (resp. an ideal) of $X$ for any $\omega \in \Omega$, then the falling shadow $\tilde{H}$ of the random set $\xi$, i.e.,

$$
\tilde{H}(x)=P(\omega \mid x \in \xi(\omega))
$$

is called a falling subalgebra (resp. falling ideal) of $X$. 
Example 2.2. Let $(\Omega, \mathscr{A}, P)$ be a probability space and let

$$
F(X):=\{f \mid f: \Omega \rightarrow X \text { is a mapping }\},
$$

where $X$ is a BCK/BCI-algebra. Define an operation $\circledast$ on $F(X)$ by

$$
(\forall \omega \in \Omega)((f \circledast g)(\omega)=f(\omega) * g(\omega))
$$

for all $f, g \in F(X)$. Let $\theta \in F(X)$ be defined by $\theta(\omega)=0$ for all $\omega \in \Omega$. It can be easily to check that $(F(X) ; \circledast, \theta)$ is a BCK/BCI-algebra. For any subalgebra/ideal $A$ of $X$ and $f \in F(X)$, let

$$
A_{f}:=\{\omega \in \Omega \mid f(\omega) \in A\}
$$

and

$$
\xi: \Omega \rightarrow \mathscr{P}(F(X)), \omega \mapsto\{f \in F(X) \mid f(\omega) \in A\} .
$$

Then $A_{f} \in \mathscr{A}$ and $\xi(\omega)=\{f \in F(X) \mid f(\omega) \in A\}$ is a subalgebra/ideal of $F(X)$. Since

$$
\xi^{-1}(\dot{f})=\{\omega \in \Omega \mid f \in \xi(\omega)\}=\{\omega \in \Omega \mid f(\omega) \in A\}=A_{f} \in \mathscr{A},
$$

$\xi$ is a random set of $F(X)$. Let

$$
\tilde{H}(f)=P(\omega \mid f(\omega) \in A) .
$$

Then $\tilde{H}$ is a falling subalgerba/ideal of $F(X)$.

Example 2.3. Let $X:=\{0, a, b, c, d\}$ be a set with the following Cayley table:

\begin{tabular}{|l|lllll|}
\hline$*$ & 0 & $a$ & $b$ & $c$ & $d$ \\
\hline 0 & 0 & 0 & 0 & 0 & 0 \\
$a$ & $a$ & 0 & 0 & $a$ & 0 \\
$b$ & $b$ & $a$ & 0 & $b$ & 0 \\
$c$ & $c$ & $c$ & $c$ & 0 & $c$ \\
$d$ & $d$ & $d$ & $d$ & $d$ & 0 \\
\hline
\end{tabular}

Then $(X ; *, 0)$ is a BCK-algebra. Let $(\Omega, \mathscr{A}, P)=([0,1], \mathscr{A}, m)$ and let $\xi:[0,1] \rightarrow \mathscr{P}(X)$ be defined by

$$
\xi(t):= \begin{cases}\{0, c\} & \text { if } t \in[0,0.3), \\ \{0, a, b, d\} & \text { if } t \in[0.3,1] .\end{cases}
$$

Then $\xi(t)$ is an ideal and hence a subalgebra of $X$ for all $t \in[0,1]$. Hence $\tilde{H}(x)=P(t \mid x \in \xi(t))$ is both a falling ideal and a falling subalgebra of 
$X$, and

$$
\tilde{H}(x)= \begin{cases}0.3 & \text { if } x=c \\ 0.7 & \text { if } x \in\{a, b, d\} \\ 1 & \text { if } x=0\end{cases}
$$

In this case, we can easily check that $\tilde{H}$ is a both fuzzy ideal and a fuzzy subalgebra of $X$.

Example 2.4. Let $X:=\{0, a, b, c\}$ be a set with the following Cayley table:

\begin{tabular}{|l|llll|}
\hline$*$ & 0 & $a$ & $b$ & $c$ \\
\hline 0 & 0 & $a$ & $b$ & $c$ \\
$a$ & $a$ & 0 & $c$ & $b$ \\
$b$ & $b$ & $c$ & 0 & $a$ \\
$c$ & $c$ & $b$ & $a$ & 0 \\
\hline
\end{tabular}

Then $(X ; *, 0)$ is a BCI-algebra. Let $(\Omega, \mathscr{A}, P)=([0,1], \mathscr{A}, m)$ and let $\xi:[0,1] \rightarrow \mathscr{P}(X)$ be defined by

$$
\xi(t):= \begin{cases}\{0, a\} & \text { if } t \in[0,0.4), \\ \{0, b\} & \text { if } t \in[0.4,0.6), \\ \{0, c\} & \text { if } t \in[0.6,1] .\end{cases}
$$

Then $\xi(t)$ is an ideal and hence a subalgebra of $X$ for all $t \in[0,1]$. Hence $\tilde{H}(x)=P(t \mid x \in \xi(t))$ is both a falling ideal and a falling subalgebra of $X$, and

$$
\tilde{H}(x)= \begin{cases}0.2 & \text { if } x=b, \\ 0.4 & \text { if } x \in\{a, c\}, \\ 1 & \text { if } x=0 .\end{cases}
$$

In this case, we know that $\tilde{H}$ is neither a fuzzy ideal nor a fuzzy subalgebra of $X$ since

$$
\begin{gathered}
\tilde{H}(b)=0.2<0.4=\min \{\tilde{H}(b * c), \tilde{H}(c)\}, \\
\tilde{H}(a * c)=\tilde{H}(b)=0.2<0.4=\min \{\tilde{H}(a), \tilde{H}(c)\} .
\end{gathered}
$$

Theorem 2.5. Let $X$ be a BCK/BCI-algebra. Then every fuzzy ideal (resp. fuzzy subalgebra) of $X$ is a falling ideal (resp. falling subalgebra) of $X$. 
Proof. Let $\tilde{H}$ be a fuzzy ideal (resp. fuzzy subalgebra) of $X$. Then $\tilde{H}_{t}$ is an ideal (resp. subalgebra) of $X$ for all $t \in[0,1]$. Let

$$
\xi:[0,1] \rightarrow \mathscr{P}(X)
$$

be a random set and $\xi(t)=\tilde{H}_{t}$. Then $\tilde{H}$ is a falling ideal (resp. falling subalgebra) of $X$.

Example 2.4 shows that the converse of Theorem 2.5 is not true in general.

Corollary 2.6. Let $X$ be a BCK-algebra. Then every falling ideal of $X$ is a falling subalgebra of $X$.

Corollary 2.6 is not valid in a BCI-algebra as seen in the following example.

Example 2.7. Let $X:=\mathbb{Q}^{*}$ be the set of all nonzero rational numbers. Let $\div$ be a binary operation on $X$ defined as division as general. Then $(X ; \div, 1)$ is a BCI-algebra (see [1]). Consider $(\Omega, \mathscr{A}, P)=$ $([0,1], \mathscr{A}, m)$ and let $\xi:[0,1] \rightarrow \mathscr{P}(X)$ be defined by

$$
\xi(t):= \begin{cases}\mathbb{Q}^{*} & \text { if } t \in[0.7,1], \\ \mathbb{Z}^{*} & \text { if } t \in[0,0.7]\end{cases}
$$

where $\mathbb{Z}^{*}$ is the set of all nonzero integers. Then $\xi(t)$ is an ideal of $X$ for all $t \in[0,1]$. Hence $\tilde{H}(x)=P(t \mid x \in \xi(t))$ is a falling ideal of $X$. But it is not a falling subalgebra of $X$ since $\xi(0.4)=\mathbb{Z}^{*}$ is not a subalgebra of $X$.

We give a condition for a falling subalgebra to be a falling ideal in a BCI-algebra.

Theorem 2.8. Let $X$ be a BCI-algebra. Assume that the falling shadow $\tilde{H}$ of a random set $\xi: \Omega \rightarrow \mathscr{P}(X)$ is a falling subalgebra of $X$. Then $\tilde{H}$ is a falling ideal of $X$ if and only if for each $\omega \in \Omega$, the following is valid:

$$
(\forall x \in \xi(\omega))(\forall y \in X \backslash \xi(\omega))(y * x \in X \backslash \xi(\omega)) .
$$

Proof. If $\tilde{H}$ is a falling ideal of $X$, then $\xi(\omega)$ is an ideal of $X$ for all $\omega \in \Omega$. Let $x, y \in X$ be such that $x \in \xi(\omega)$ and $y \in X \backslash \xi(\omega)$. If $y * x \in \xi(\omega)$, then $y \in \xi(\omega)$ which is a contradiction. Hence (2.2) is valid. Conversely, let $\tilde{H}$ be a falling subalgebra of $X$ that satisfies (2.2). Then $\xi(\omega)$ is a subalgebra of $X$ for $\omega \in \Omega$. Hence $0 \in \xi(\omega)$. Let $x, y \in X$ be such that $x * y \in \xi(\omega)$ and $y \in \xi(\omega)$. If $x \notin \xi(\omega)$, then $x * y \in X \backslash \xi(\omega)$ by (2.2). This is a contradiction, and so $\tilde{H}$ is a falling ideal of $X$. 
Let $X$ be a BCK/BCI-algebra and $(\Omega, \mathscr{A}, P)$ a probability space. Let $\tilde{H}$ be a falling shadow of a random set $\xi: \Omega \rightarrow \mathscr{P}(X)$. For $x \in X$, let

$$
\Omega(x ; \xi):=\{\omega \in \Omega \mid x \in \xi(\omega)\} .
$$

Then $\Omega(x ; \xi) \in \mathscr{A}$.

Proposition 2.9. If $\tilde{H}$ is a falling ideal of a $B C K / B C I$-algebra $X$, then

$$
\begin{gathered}
(\forall x, y \in X)(x \leq y \Longrightarrow \Omega(y ; \xi) \subseteq \Omega(x ; \xi)), \\
(\forall x, y \in X)(\Omega(x * y ; \xi) \cap \Omega(y ; \xi) \subseteq \Omega(x ; \xi)) .
\end{gathered}
$$

If $\tilde{H}$ is a falling subalgebra of a $B C K / B C I$-algebra $X$, then

$$
(\forall x, y \in X)(\Omega(x ; \xi) \cap \Omega(y ; \xi) \subseteq \Omega(x * y ; \xi)) .
$$

If $\tilde{H}$ is a falling subalgebra/ideal of a BCK-algebra $X$, then

$$
(\forall x \in X)(\Omega(x ; \xi) \subseteq \Omega(0 ; \xi)) .
$$

If $\tilde{H}$ is a falling ideal of a BCK-algebra $X$, then

$$
(\forall x, y \in X)(\Omega(x ; \xi) \subseteq \Omega(x * y ; \xi)) .
$$

Proof. Let $x, y \in X$ be such that $x \leq y$ and let $w \in \Omega(y ; \xi)$. Then $y \in$ $\xi(\omega)$ and $x * y=0 \in \xi(\omega)$. It follows that $x \in \xi(\omega)$ so that $\omega \in \Omega(x ; \xi)$. Hence (2.4) is valid. Let $w \in \Omega(x * y ; \xi) \cap \Omega(y ; \xi)$. Then $x * y \in \xi(\omega)$ and $y \in \xi(\omega)$. Since $\xi(\omega)$ is an ideal of $X$, it follows that $x \in \xi(\omega)$ so that $w \in \Omega(x ; \xi)$. This shows that (2.5) is satisfied. If $\omega \in \Omega(x ; \xi) \cap \Omega(y ; \xi)$, then $x \in \xi(\omega)$ and $y \in \xi(\omega)$. Since $\xi(\omega)$ is a subalgebra of $X$, we have $x * y \in \xi(\omega)$ and hence $\omega \in \Omega(x * y ; \xi)$. Thus (2.6) is valid. Since $0 \leq x$ for all $x \in X$, the result (2.7) follows from (2.4). Let $\omega \in \Omega(x ; \xi)$. Then $x \in \xi(\omega)$. Since $x * y \leq x$ for all $x, y \in X$ and since $\xi(\omega)$ is an ideal of $X$, it follows from (1.1) that $x * y \in \xi(\omega)$ so that $\Omega(x ; \xi) \subseteq \Omega(x * y ; \xi)$.

Theorem 2.10. If $\tilde{H}$ is a falling subalgebra of a $B C K / B C I$-algebra $X$, then

$$
(\forall x, y \in X)\left(\tilde{H}(x * y) \geq T_{m}(\tilde{H}(x), \tilde{H}(y))\right)
$$

where $T_{m}(s, t)=\max \{s+t-1,0\}$ for any $s, t \in[0,1]$. Hence

Proof. By Definition 2.1, $\xi(\omega)$ is a subalgebra of $X$ for any $\omega \in \Omega$.

$$
\{\omega \in \Omega \mid x \in \xi(\omega)\} \cap\{\omega \in \Omega \mid y \in \xi(\omega)\} \subseteq\{\omega \in \Omega \mid x * y \in \xi(\omega)\},
$$


and so

$$
\begin{aligned}
\tilde{H}(x * y) & =P(\omega \mid x * y \in \xi(\omega)) \\
\geq & P(\{\omega \mid x \in \xi(\omega)\} \cap\{\omega \mid y \in \xi(\omega)\}) \\
\geq & P(\omega \mid x \in \xi(\omega))+P(\omega \mid y \in \xi(\omega)) \\
& \quad-P(\omega \mid x \in \xi(\omega) \text { or } y \in \xi(\omega)) \\
\geq & \tilde{H}(x)+\tilde{H}(y)-1 .
\end{aligned}
$$

Hence

$$
\tilde{H}(x * y) \geq \max \{\tilde{H}(x)+\tilde{H}(y)-1,0\}=T_{m}(\tilde{H}(x), \tilde{H}(y)) .
$$

This completes the proof.

Theorem 2.10 means that every falling subalgebra of a BCK/BCIalgebra $X$ is a $T_{m}$-fuzzy subalgebra of $X$.

Theorem 2.11. If $\tilde{H}$ is a falling ideal of a $B C K / B C I$-algebra $X$, then

$$
(\forall x, y \in X)\left(\tilde{H}(x) \geq T_{m}(\tilde{H}(x * y), \tilde{H}(y))\right)
$$

where $T_{m}(s, t)=\max \{s+t-1,0\}$ for any $s, t \in[0,1]$.

Proof. By Definition 2.1, $\xi(\omega)$ is an ideal of $X$ for any $\omega \in \Omega$. Hence

$$
\{\omega \in \Omega \mid x * y \in \xi(\omega)\} \cap\{\omega \in \Omega \mid y \in \xi(\omega)\} \subseteq\{\omega \in \Omega \mid x \in \xi(\omega)\},
$$

and thus

$$
\begin{aligned}
\tilde{H}(x) & =P(\omega \mid x \in \xi(\omega)) \\
& \geq P(\{\omega \mid x * y \in \xi(\omega)\} \cap\{\omega \mid y \in \xi(\omega)\}) \\
& \geq P(\omega \mid x * y \in \xi(\omega))+P(\omega \mid y \in \xi(\omega)) \\
& \quad-P(\omega \mid x * y \in \xi(\omega) \text { or } \omega \mid y \in \xi(\omega)) \\
& \geq \tilde{H}(x * y)+\tilde{H}(y)-1 .
\end{aligned}
$$

Hence

$$
\tilde{H}(x) \geq \max \{\tilde{H}(x * y)+\tilde{H}(y)-1,0\}=T_{m}(\tilde{H}(x * y), \tilde{H}(y)) .
$$

This completes the proof.

Theorem 2.11 means that every falling ideal of a BCK/BCI-algebra $X$ is a $T_{m}$-fuzzy ideal of $X$. 


\section{Conclusion}

Falling shadow representation theory shows us the way of selection relaid on the joint degrees distributions. It is reasonable and convenient approach for the theoretical development and the practical applications of fuzzy sets and fuzzy logics. The theory of falling shadows relates probability concepts with the membership functions of fuzzy sets. As an algebraic approach of the theory of falling shadows, Yuan and Lee [9] have considered a fuzzy subgroup (subring, ideal) as the falling shadow of the cloud of the subgroup (subring, ideal). In this paper, we discussed the notion of falling subalgebras/ideals in BCK/BCI-algebras based on the theory of falling shadows. We gave relations between falling subalgebras and falling ideals. We also provided relations between fuzzy subalgebras/ideals and falling subalgebras/ideals. We established a characterization of a falling ideal, and showed that every falling subalgebra/ideal is a $T_{m}$-fuzzy subalgebra/ideal. Based on these results, we will apply the theory of falling shadows to the other type of ideals in BCK/BCIalgebras in the future study.

\section{References}

[1] Z. M. Chen and H. X. Wang, On ideals in BCI-algebras. Math. Japon. 36(3) (1991), 497-501.

[2] I. R. Goodman, Fuzzy sets as equivalence classes of random sets, in "Recent Develop- ments in Fuzzy Sets and Possibility Theory" (R. Yager, Ed.), Pergamon, New York 1982, pp. 327-343.

[3] K. Iséki and S. Tanaka, An introduction to the theory of BCK-algebras, Math. Jpn. 23 (1978), 1-26.

[4] J. Meng and Y. B. Jun, BCK-algebras, Kyungmoon Sa Co. Seoul 1994.

[5] S. K. Tan, P. Z. Wang and X. Z. Zhang, Fuzzy inference relation based on the theory of falling shadows, Fuzzy Sets and Systems 53 (1993), 179-188.

[6] S. K. Tan, P. Z. Wang and E. S. Lee, Fuzzy set operations based on the theory of falling shadows, J. Math. Anal. Appl. 174 (1993), 242-55.

[7] P. Z. Wang, Fuzzy Sets and Falling Shadows of Random Sets, Beijing Normal Univ. Press, People's Republic of China 1985. [In Chinese]

[8] P. Z. Wang and E. Sanchez, Treating a fuzzy subset as a projectable random set, in "Fuzzy Information and Decision" (M. M. Gupta and E. Sanchez, Eds.), Pergamon, New York 1982, pp. 212-219.

[9] X. H. Yuan and E. S. Lee, A fuzzy algebraic system based on the theory of falling shadows, J. Math. Anal. Appl. 208 (1997), 243-251. 
Young Bae Jun

Department of Mathematics Education (and RINS), Gyeongsang National University,

Chinju 660-701, Korea.

E-mail: skywine@gmail.com

Chul Hwan Park

School of Digital,Mechanics, Ulsan College,

Ulsan 680-749, Korea.

E-mail: skyrosemary@gmail.com 